Louisiana State University

LSU Digital Commons

8-5-2008

\title{
A detailed FLUKA-2005 Monte-Carlo simulation for the ATIC detector
}

\author{
R. M. Gunasingha \\ Southern University and A\&M College
}

A. R. Fazely

Southern University and A\&M College

J. H. Adams

NASA Marshall Space Flight Center

H. S. Ahn

University of Maryland, College Park

G. L. Bashindzhagyan

Lomonosov Moscow State University

See next page for additional authors

Follow this and additional works at: https://digitalcommons.Isu.edu/physics_astronomy_pubs

\section{Recommended Citation}

Gunasingha, R., Fazely, A., Adams, J., Ahn, H., Bashindzhagyan, G., Batkov, K., Chang, J., Christl, M., Ganel, O., Guzik, T., Isbert, J., Kim, K., Kouznetsov, E., Panasyuk, M., Panov, A., Schmidt, W., Seo, E., Sokolskaya, N., Watts, J., Wefel, J., Wu, J., Zatsepin, V., \& Lin, Z. (2008). A detailed FLUKA-2005 Monte-Carlo simulation for the ATIC detector. Advances in Space Research, 42 (3), 417-423. https://doi.org/10.1016/

j.asr.2007.03.079

This Article is brought to you for free and open access by the Department of Physics \& Astronomy at LSU Digital Commons. It has been accepted for inclusion in Faculty Publications by an authorized administrator of LSU Digital Commons. For more information, please contact ir@lsu.edu. 


\section{Authors}

R. M. Gunasingha, A. R. Fazely, J. H. Adams, H. S. Ahn, G. L. Bashindzhagyan, K. E. Batkov, J. Chang, M. Christl, O. Ganel, T. G. Guzik, J. Isbert, K. C. Kim, E. N. Kouznetsov, M. I. Panasyuk, A. D. Panov, W. K.H. Schmidt, E. S. Seo, N. V. Sokolskaya, J. W. Watts, J. P. Wefel, J. Wu, V. I. Zatsepin, and Z. W. Lin 


\title{
A Detailed Fluka-2005 Monte Carlo Simulation for the ATIC Detector
}

\author{
R. M. Gunasingha ${ }^{a, *}$ A. R. Fazely ${ }^{a}$ J. H. Adams, Jr. ${ }^{b}$
}

H. S. Ahn ${ }^{\text {c }}$ G. L. Bashindzhagyan ${ }^{\text {d }}$ K. E. Batkov ${ }^{\text {c }}$ J. Chang e,1

M. Christl ${ }^{\mathrm{b}}$ O. Ganel ${ }^{\mathrm{c}}$ T. G. Guzik ${ }^{\mathrm{f}}$ J. Isbert $^{\mathrm{f}}$ K. C. Kim ${ }^{\mathrm{c}}$ E. N. Kouznetsov ${ }^{d}$ M. I. Panasyuk ${ }^{d}$ A. D. Panov ${ }^{d}$

W. K. H. Schmidt ${ }^{e}$ E. S. Seo ${ }^{c}$ N. V. Sokolskaya ${ }^{d}$ J. W. Watts ${ }^{b}$

J. P. Wefel ${ }^{\mathrm{f}}$ J. Wu ${ }^{\mathrm{c}}$ V. I. Zatsepin ${ }^{\mathrm{d}}$ Z. W. Lin ${ }^{\mathrm{g}}$

${ }^{a}$ Southern University, Physics Department, Baton Rouge, LA, USA.

${ }^{\mathrm{b}}$ Marshall Space Flight Center, Huntsville, AL, USA

${ }^{\mathrm{c}}$ University of Maryland, Institute for Physical Science $\mathcal{E}$ Technology, College

Park, MD, USA

${ }^{\mathrm{d}}$ Skobeltsyn Institute of Nuclear Physics, Moscow State University, Moscow, Russia

${ }^{\mathrm{e}}$ Max-Plank Institute for Solar System Research, Katlenburg-Lindau, Germany

${ }^{\mathrm{f}}$ Louisiana State University, Department of Physics $\&$ Astronomy, Baton Rouge, $L A, U S A$

${ }^{g}$ University of Alabama, Huntsville, $A L, U S A$

\begin{abstract}
We have performed a detailed Monte Carlo (MC) simulation for the Advanced Thin Ionization Calorimeter (ATIC) detector using the MC code FLUKA-2005 which is capable of simulating particles up to $10 \mathrm{PeV}$. The ATIC detector has completed two successful balloon flights from McMurdo, Antarctica lasting a total of more than 35 days. ATIC is designed as a multiple, long duration balloon flight, investigation of the cosmic ray spectra from below $50 \mathrm{GeV}$ to near $100 \mathrm{TeV}$ total energy; using a fully active Bismuth Germanate(BGO) calorimeter. It is equipped with a large mosaic of silicon detector pixels capable of charge identification, and, for particle tracking, three projective layers of $\mathrm{x}-\mathrm{y}$ scintillator hodoscopes, located above, in the middle and below a 0.75 nuclear interaction length graphite target. Our simulations are part of an analysis package of both nuclear (A) and energy dependences for different nuclei interacting in the ATIC detector. The MC simulates the response of different components of the detector such as the Si-matrix, the scintillator hodoscopes and the BGO calorimeter to various nuclei. We present comparisons of the FLUKA-2005 MC calculations with GEANT calculations and with the ATIC CERN data.
\end{abstract}




\section{Introduction}

High energy cosmic rays, particles of matter from distant regions of our galaxy and possibly elsewhere in the universe, can be directly observed by balloonborne or space-based experiments. Understanding the cosmic ray composition and energy spectra can provide clues to the origin of cosmic rays, their acceleration mechanism, and their propagation through the galactic and intergalactic media. Cosmic rays contain all natural elements from proton to nuclei beyond nickel. The all-particle spectrum of cosmic rays (where species can not be distinguished) obeys a power law dependence of the observed flux on energy from $10 \mathrm{GeV}$ up to the highest energies measured by ground-based air shower array experiments, over $10^{20} \mathrm{eV}$. From these measurements, it is known that the spectrum is somewhat steeper above $10^{16} \mathrm{eV}$ than it is below $10^{14} \mathrm{eV}$. This steepening is called the "knee". It is not known whether this knee is due to:

- a change in the propagation of cosmic rays;

- a change in the acceleration mechanism of cosmic rays;

- a change in the composition of the cosmic rays;

The Advanced Thin Ionization Calorimeter (ATIC) is a balloon-borne experiment designed to investigate the elemental spectra of protons and other nuclei up to $Z=26$, for energies from $50 \mathrm{GeV}$ to $100 \mathrm{TeV}$, with a statistical accuracy of better than $30 \%$ for protons at the highest energy.(1) The ATIC balloon payload was launched to an altitude of $36 \mathrm{~km}$ above sea level from McMurdo, Antarctica in December 2000 and December 2002. These two flights lasted more than 35 days.

In this paper, we discuss a data analysis tool we have developed based on FLUKA-2005. This code provides the capability of simulating high energy intercations up to $100 \mathrm{TeV}$, the highest energy measured by the ATIC detector.

\footnotetext{
* Corresponding author. Email address: phguna@feynman.phys.subr.edu (R. M. Gunasingha). URL: http://suphys.phys.subr.edu (R. M. Gunasingha).

1 Present address: Purple Mountain Observatory, Chinese Academy of Sciences, China
} 


\section{Scientific Objectives}

The ATIC detector focuses on direct measurements of high energy cosmic rays. The main scientific objectives of the ATIC experiment are:

- to measure the $\mathrm{H}$ and He energy spectrum from $\approx 10^{10} \mathrm{eV}$ to $\approx 10^{14} \mathrm{eV}$ with a single instrument;

- to investigate the spectral differences between protons and helium;

- to determine if the proton spectrum has a bend between $2 \mathrm{TeV}$ and $\approx 40$ $\mathrm{TeV}$;

- to accurately measure the $\mathrm{H} / \mathrm{He}$ ratio as a function of energy;

- to determine if the spectra of heavy nuclei have the same indices;

- to search for high-energy electrons and neutrals;

\section{$3 \quad$ ATIC Detector}

The ATIC detector shown in figure 1 is comprised of a fully active $50 \mathrm{~cm} \times$ $50 \mathrm{~cm} \times 20 \mathrm{~cm}$ deep BGO calorimeter, preceded by three $10 \mathrm{~cm}$ thick graphite targets sandwiched between panels of scintillator hodoscopes, having an outwardly projective angle of 24 degrees. It is equipped at the top with a large mosaic of silicon detector pixels capable of charge identification. The BGO crystals of dimensions $2.5 \mathrm{~cm} \times 2.5 \mathrm{~cm} \times 25.0 \mathrm{~cm}$ long are arranged horizontally in 8 layers, with the long axis aligned alternaltely along the $\mathrm{X}$ and $\mathrm{Y}$ axes. The vertical direction is along the $\mathrm{Z}$ axis. There are 320 BGO crystals with 40 crystals in each layer. The construction and commissioning of the detector, the data acquisition system and the software development for the detector, and detector calibration are discussed elsewhere (1).

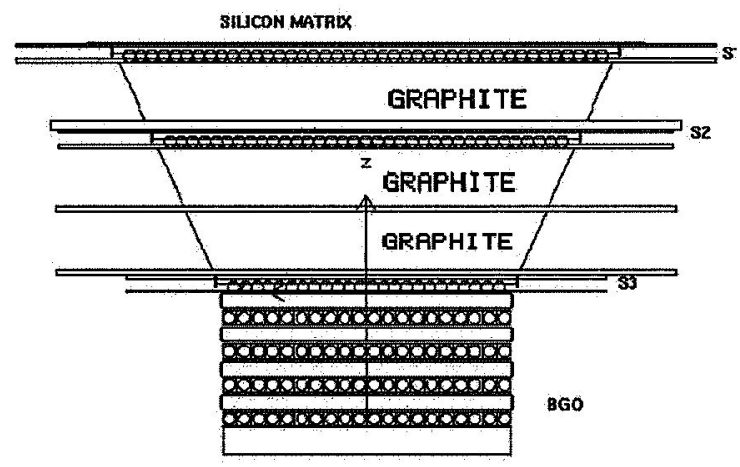

Fig. 1. ATIC detector, showing the Si-matrix, the three scintillator hodoscopes S1, S2, and S3, the graphite targets and the BGO calorimeter. 


\section{GEANT-3.21 ATIC Monte Carlo}

We have written a MC code for the ATIC detector using GEANT-3.21, that generates events with energies less than $20 \mathrm{TeV}$. Note that GEANT-3.21 is presently limited to energies below $20 \mathrm{TeV}$ for hadrons. Figure 1 was generated by GEANT-3.21 using a geometry input file for the ATIC detector.

In 1999, the ATIC detector was calibrated using test beams at CERN. The detector was illuminated with $375 \mathrm{GeV}$ protons, $300 \mathrm{GeV}$ electrons, $150 \mathrm{GeV}$ electrons and $150 \mathrm{GeV}$ pions. Figure 2 shows the energy spectra from data and $\mathrm{MC}$ simulations for $375 \mathrm{GeV}$ protons. The data and the simulated results agreed well as expected. Figures 3 and 4 show the comparison of data for 150 $\mathrm{GeV}$ protons and pions with our GEANT3.21 MC calculations. Note the MC calculations have been performed for the same number of events as the data.

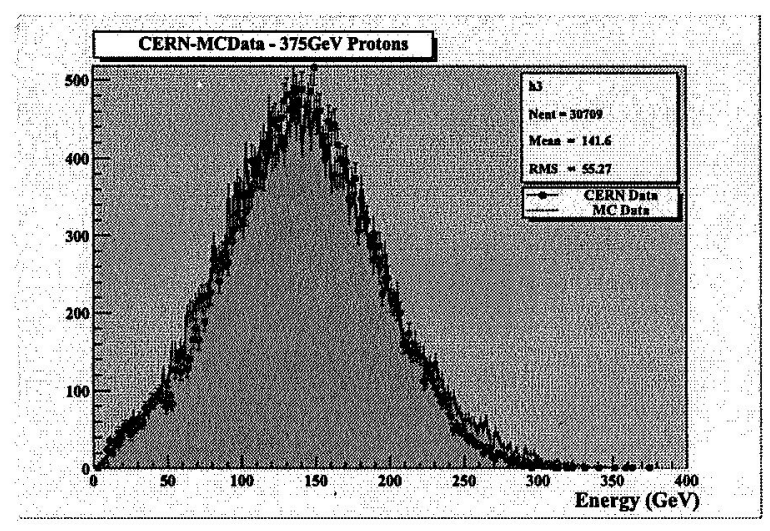

Fig. 2. Comparison of CERN $375 \mathrm{GeV}$ proton data with GEANT.

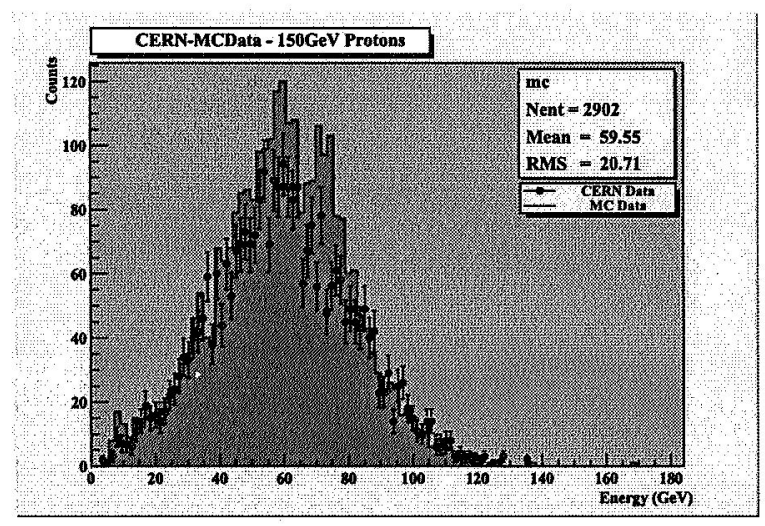

Fig. 3. Comparison of CERN $150 \mathrm{GeV}$ proton data with GEANT.

GEANT is widely used in high energy physics for the simulation of primary particles such as $\mathrm{p}$, e and $\mu$. When GEANT4 was introduced the support for GEANT-3.21 became limited. GEANT4 is evolving very rapidly but it still 


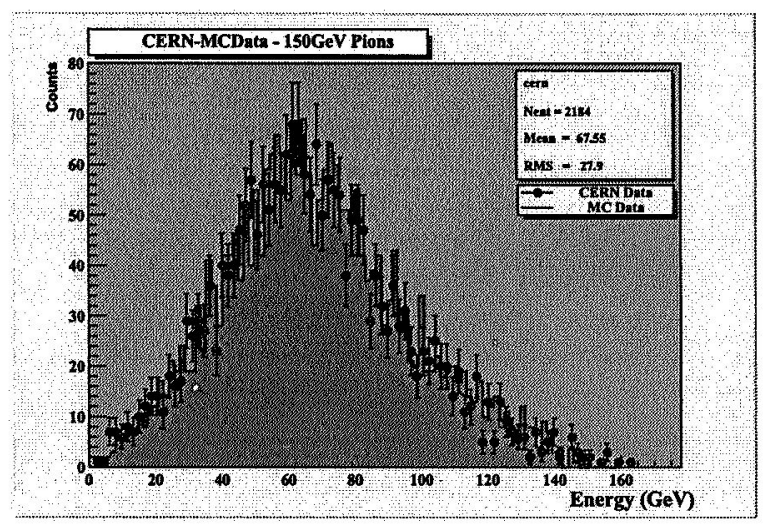

Fig. 4. Comparison of CERN $150 \mathrm{GeV}$ pion data with GEANT.

has some limitations for simulations at very high energies ( $\mathrm{TeV}$ to $\mathrm{PeV}$ range) and cannot provide simulations of heavy ions.

One of the primary goals of ATIC is to determine the indices for the energy spectrum of heavy ions from $\mathrm{He}, \mathrm{C}, \mathrm{N}, \mathrm{O}$ to $\mathrm{Fe}$ up to very high energies. For this purpose we had to find a reliable simulation code to simulate heavy ions. After a thorough search, we decided to use FLUKA.

\section{Description of the FLUKA Code}

FLUKA which stands for "FLUktuierende KAskade" is a MC code able to simulate the transport and interaction of hadronic and electromagnetic particles on any target material. It is a multi-particle, multipurpose code and is used in many different fields such as medical physics, astrophysics and high energy physics (2). It has been tested and verified with real data. For light nuclei, it uses the Fermi break model. Above $5 \mathrm{GeV} /$ nucleon, the Dual Parton Model (DPM) is used. FLUKA can treat combinatorial geometry. It is widely used for studies related to both basic research and to applications in radiation propagation, dosimetry, cosmic ray and accelerator physics. Followings are a few areas to which modern FLUKA has been successfully applied (3).

- Accelerators and shielding - CERN, SLAC;

- Background and radiation damage studies- ATLAS and LHC experiments;

- Dosimetry and radiotherapy- NASA;

- Calorimetry - ATLAS test beams and ICARUS neutrino experiment;

One of the main reasons for using FLUKA for ATIC is the ability to transport heavy ions at very high energies. FLUKA is designed to transport heavy ions and their interaction products from $5 \mathrm{GeV} /$ nucleon to very high energies, approximately $10 \mathrm{PeV}$, using a DPMJET-3 code which is a high energy 
hadron-hadron, hadron-nucleus, nucleus-nucleus interaction model. It can run on various platforms such as Linux, Unix and HP. The validity of the models implemented in FLUKA have been benchmarked against several experimental data, from accelerators to cosmic ray showers (2). The FLUKA code is maintained and updated regularly. The FLUKA discussion archive provides much support for the FLUKA user community (4).

\section{THe ATIC-FLUKA Code}

We implemented the ATIC geometry in the FLUKA code. Events were randomly generated in position, direction and energy above the Si-matrix plane. An energy dependence of $E^{-2.7}$ was assumed. Figure 5 shows a $150 \mathrm{GeV}$ proton vertically incident on ATIC very close to the center on top of the detector.

$150 \mathrm{GeV}$ proton ATIC-FLUKA

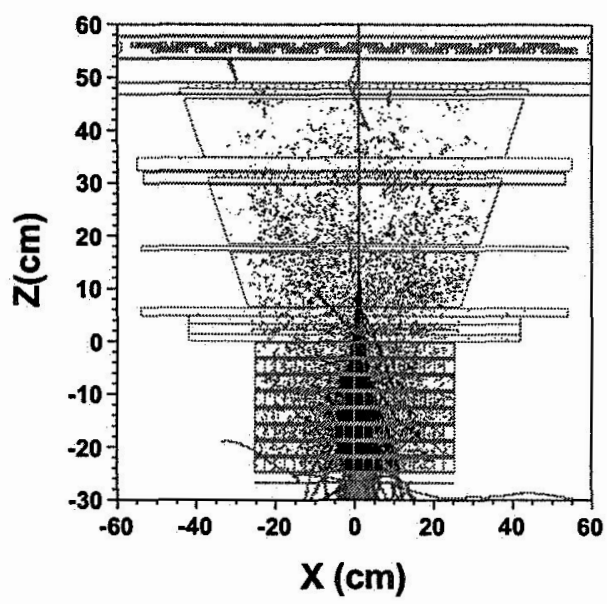

Fig. 5. A $150 \mathrm{GeV}$ proton interacting in the ATIC detector

We have compared the energy deposition in the ATIC BGO calorimeter using GEANT-3.21 and FLUKA. Figure 6 shows this comparison. Figure 7 shows the comparison of energy deposition by $1 \mathrm{TeV}$ protons in ATIC. Figure 8 shows the energy distributions obtained from FLUKA and CERN data for $375 \mathrm{GeV}$ protons incident on ATIC. Although at lower enrgies the agreemnet between GEANT and FLUKA and CERN data are good at higher energy, namely $1 \mathrm{TeV}$, there is a 10\% descrepancy between GEANT and FLUKA. Because there is no data avaible at $1.0 \mathrm{TeV}$ for ATIC, it is not clear which simulation is better. 


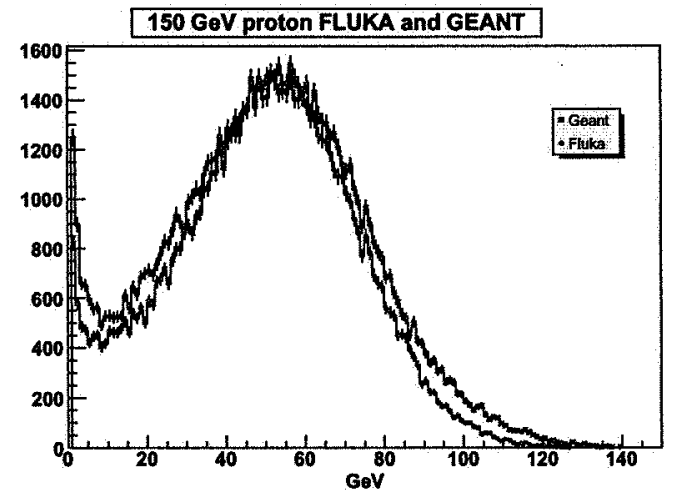

Fig. 6. Comparison of the deposited energy spectra in the BGO calorimeter for 150 $\mathrm{GeV}$ protons from GEANT and FLUKA calculations

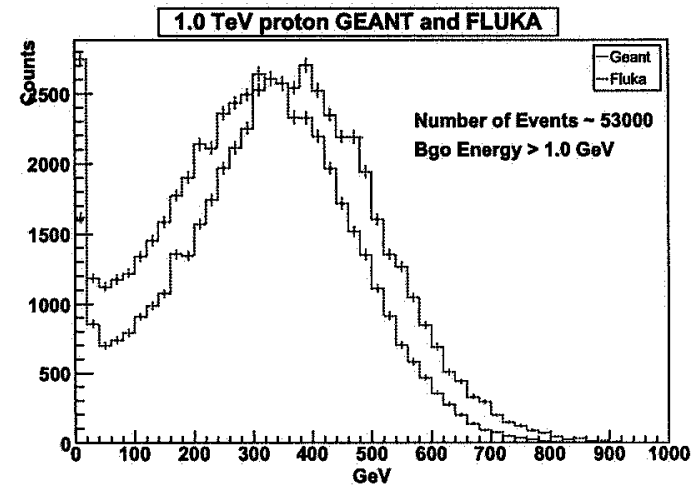

Fig. 7. Comparison of the deposited energy spectra in the BGO calorimeter for 1.0 $\mathrm{TeV}$ protons from GEANT and FLUKA calculations

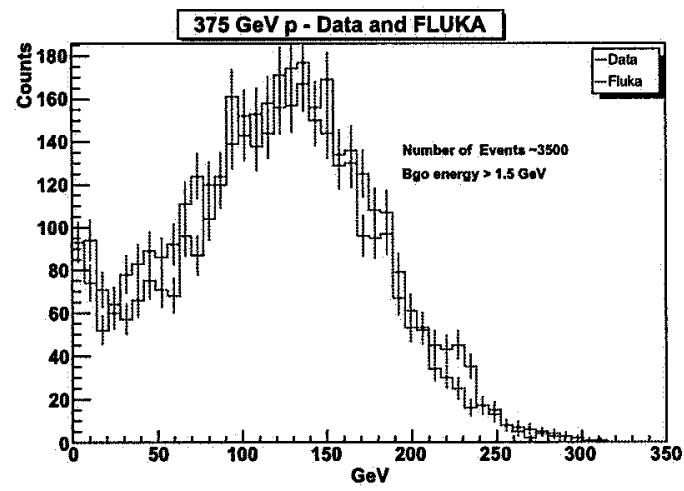

Fig. 8. Comparison of the deposited energy spectra in the BGO calorimeter for 375 $\mathrm{GeV}$ protons from CERN data and FLUKA calculations

\section{Heavy Ion Simulation}

One of the primary goals of ATIC is to determine the indices for the energy spectra of nuclei such as $\mathrm{He}, \mathrm{C}, \mathrm{N}, \mathrm{O}$ up to Fe. For this purpose, we implemented 
the DPMJET-3 interface to the FLUKA input card file. Figure 9 shows a 12 $\mathrm{TeV}{ }^{12} C$ nucleus vertically incident on ATIC close to the center of the detector.

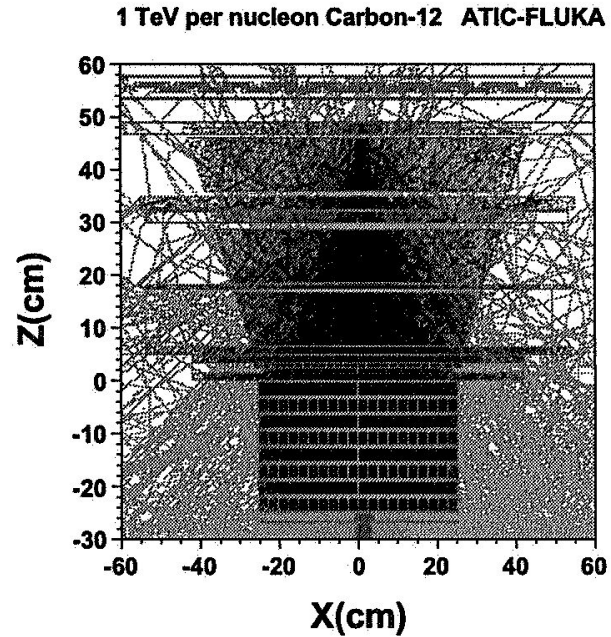

Fig. 9. A $12 \mathrm{TeV}^{12} \mathrm{C}$ nucleus interacting in the ATIC detector

Figure 10 shows an event display for a $40 \mathrm{TeV}{ }^{14} \mathrm{~N}$ nucleus incident on ATIC with a randomly chosen position and direction. Figure 11 shows a $64 \mathrm{TeV}^{16} \mathrm{O}$ nucleus entering the top of the detector with a randomly chosen position and direction.

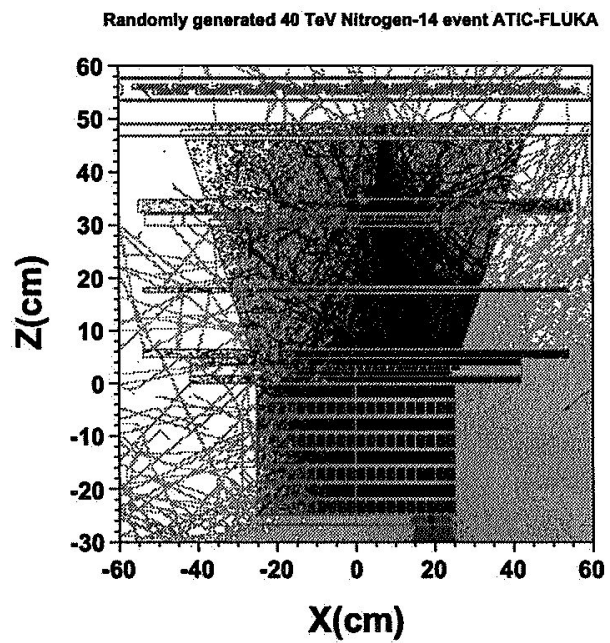

Fig. 10. A $40 \mathrm{TeV}{ }^{14} N$ nucleus interacting in the ATIC detector 


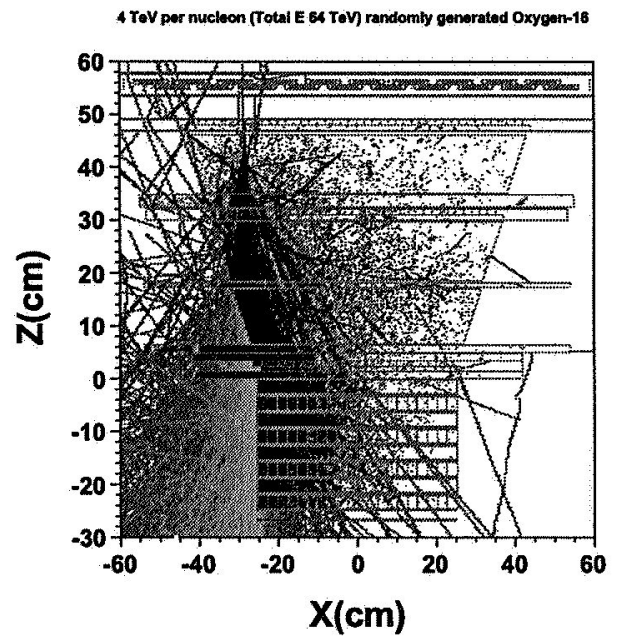

Fig. 11. A $64 \mathrm{TeV}{ }^{16} \mathrm{O}$ nucleus interacting in the ATIC detector

\section{Event Generation and Reconstruction}

An important goal of the simulation for the ATIC detector is to calculate efficiencies for various cuts applied to the data, so that an absolute cosmic ray flux can be obtained. The spectra of cosmic rays is well described by an inverse power law in energy with differential flux given by $\frac{d n}{d E}=C E^{-2.70}$. We have written a user code to generate events according to the above power law with energies ranging from $10 \mathrm{GeV}$ to $100 \mathrm{TeV}$. The position is randomly chosen to be between $-40 \mathrm{~cm}$ and $+40 \mathrm{~cm}$ in both $\mathrm{x}$ and $\mathrm{y}$. All events were generated in a plane at $10 \mathrm{~cm}$ above the top of the detector. The spectra of generated events is shown in figure 12 .

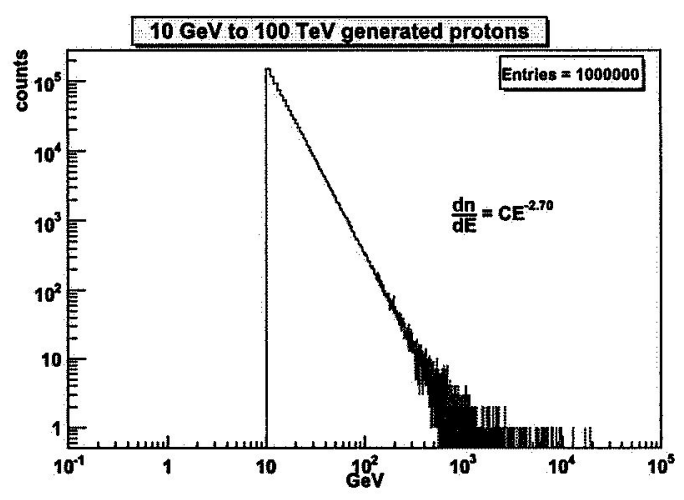

Fig. 12. $10 \mathrm{GeV}$ to $100 \mathrm{TeV}$ randomly generated protons on ATIC using FLUKA

We ran the ATIC-FLIKA MC for 1,000,000 protons with energies ranging 
from $50 \mathrm{GeV}$ to $100 \mathrm{TeV}$. After applying a fiducial volume cut on the particle trajectory $2.5 \mathrm{~cm}$ from the edge of the calorimeter in $\mathrm{x}$ and $\mathrm{y}, 250,000$ events remained. Figure 13 shows the distribution of the energy deposited in the BGO calorimeter for the events passing the fiducial volume cut. The resulting MC generated data are in the same format as the actual data and can be analyzed in the same manner.

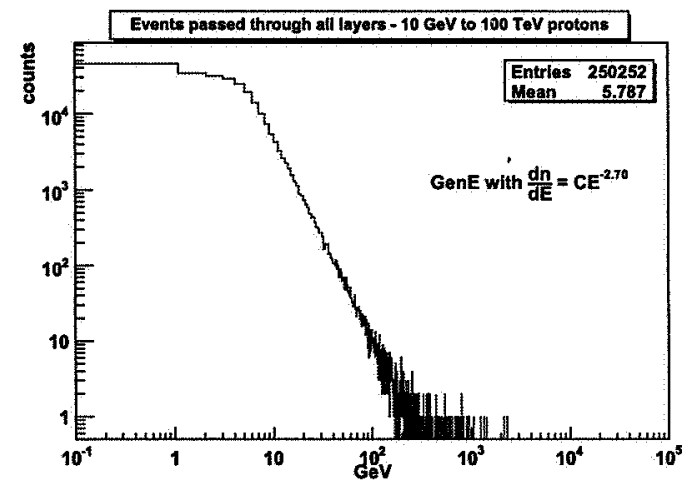

Fig. 13. Energy deposited in the BGO calorimeter by events staifying the fiducial volume cut

To insure the integrity of trajectory reconstruction, we required that every BGO plane satisfy an energy threshold selection of $1.0 \mathrm{GeV}$. The energy distribution of such events is shown in figure 14.

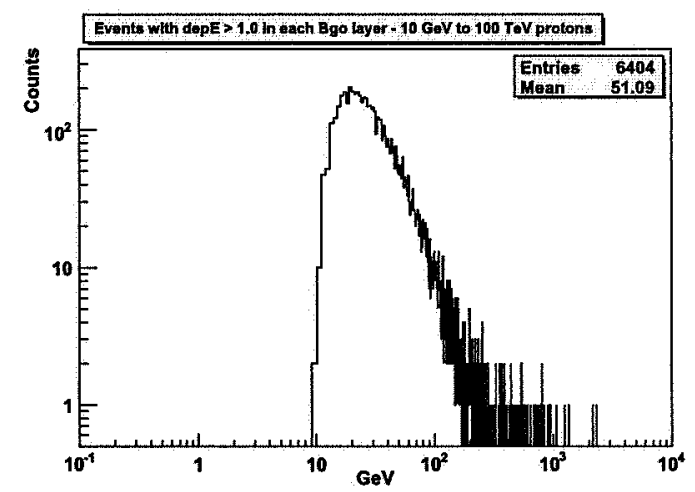

Fig. 14. The energy of the events with a mininum deposited energy of $1.0 \mathrm{GeV}$ in each layer of BGO.

The reconstruction of the trajectory was obtained from a $\chi^{2}$ fit using the weighted pulse-heights in the BGO crystals. The trajectory was them improved using the position of the hit pixel in the Si-matrix plane as well as any hit scintillator along its intersection with the hodoscope. To check the goodness of the fit, we extended the trajectory back to the plane where the MC events were generated. The distance between the generated points and the reconstructed points is shown in Figure 15. 


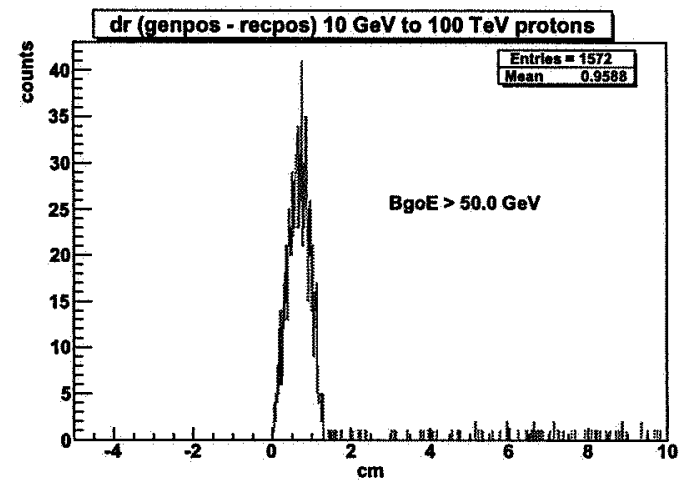

Fig. 15. The distance between the reconstructed positions and the generated positions on a plane $10 \mathrm{~cm}$ above the Si-matrix

For the ${ }^{12} C$ simulation, a total of 550,000 events out of $1,380,000$ events passed the above-mentioned fiducial volume cut. Figures 16, 17, and 18 show generated, accepted and threshold selected energies, respectively. Figure 19 shows the distance between reconstructed and generated positions.

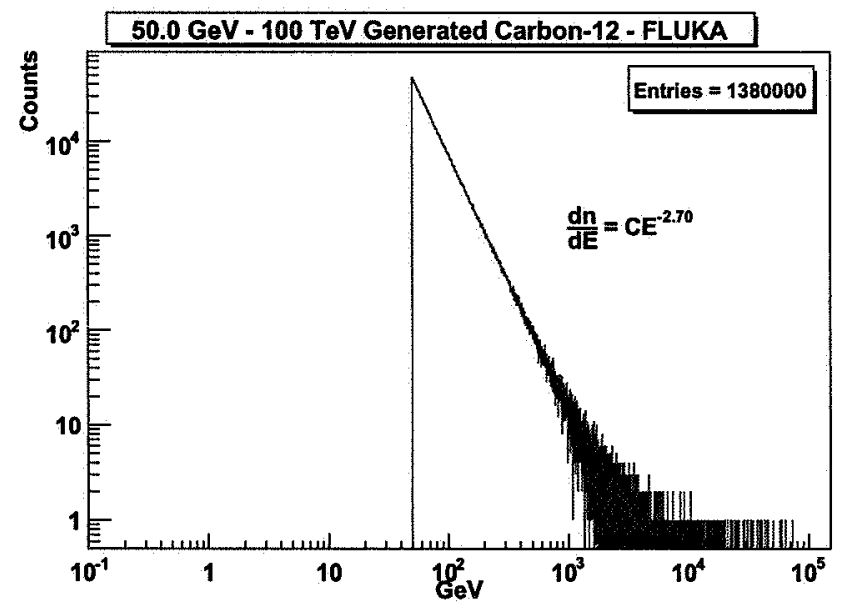

Fig. $16.50 \mathrm{GeV}$ to $100 \mathrm{TeV}$ randomly generated ${ }^{12} C$ events

\section{Conclusion}

A simulation code was developed for the ATIC detector using FLUKA-2005. This code can be used to simulate ATIC events for very high energy primary particles ( $p, e$, and $\mu$ etc.) and heavy ions. The FLUKA-2005 code provides simulation of particles to the maximum energy $\approx 100 \mathrm{TeV}$ measured by ATIC. We compared MC data from standard GEANT-3.21 and FLUKA below 20 $\mathrm{TeV}$. The agreement is reasonable. This ATIC-FLUKA MC code provides a 


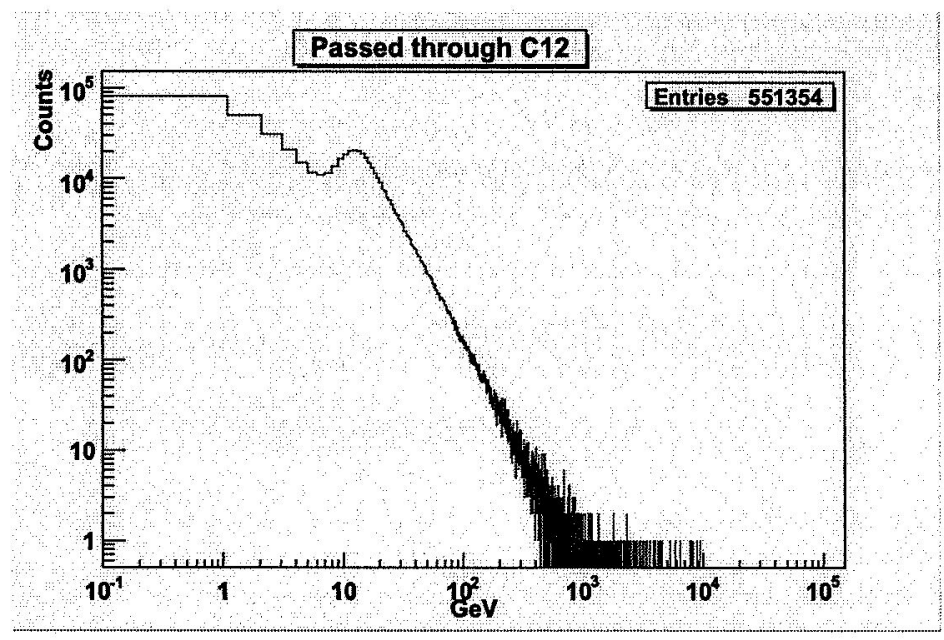

Fig. 17. Energy deposited in the BGO calorimeter by ${ }^{12} \mathrm{C}$ events staifying the fiducial volume cut

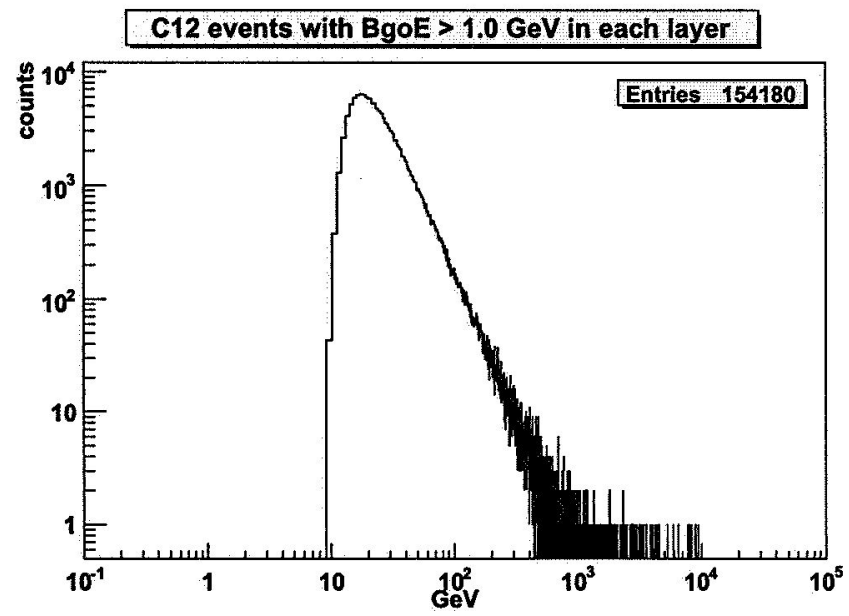

Fig. 18. The energy deposition of ${ }^{12} C$ events where each layer has more than 1.0 $\mathrm{GeV}$ energy deposition.

pivotal role for the analysis of the ATIC data and will be a part of the analysis package for heavy ions at high energies, not achievable by the GEANT-3.21 MC.

Acknowledgments

We like to thank Richard L. Imlay for many wondeful discussions regarding this paper. This work was supported in the United States by a grant from the National Aeronautics and Space Administration (NASA), to Southern University under the grant number NNGO4WC06G. 


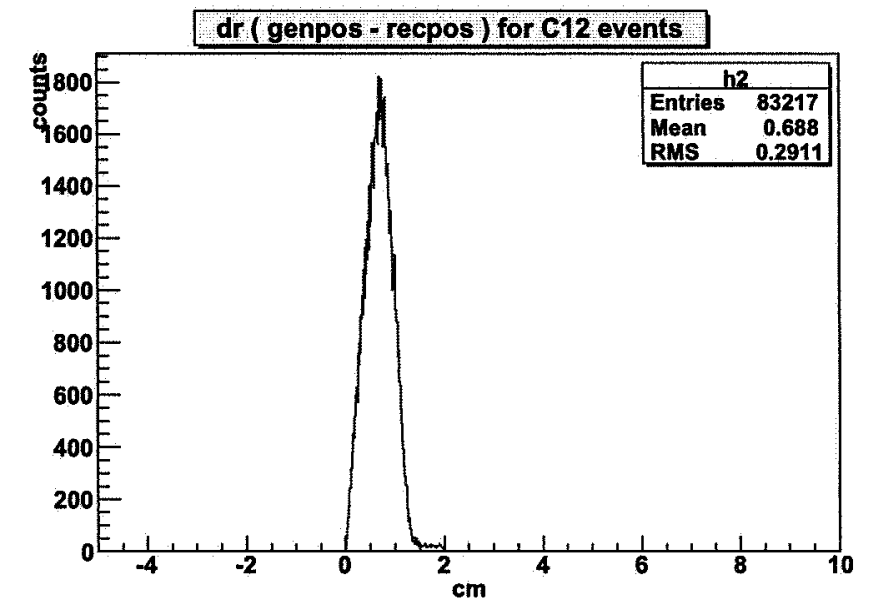

Fig. 19. The distance between the generated position and the reconstructed positions for $50 \mathrm{GeV}$ to $100 \mathrm{TeV}$ randomly generated ${ }^{12} C$ events.

\section{References}

[1] Guzik T. G. et. al., The Advanced Thin Ionization Calorimeter (ATIC) for studies of High Energy Cosmic Rays, in proceedings of the 26th ICRC, eds. D. Kieda, M. Salamon and B. Dingus, 5, pp 9-12, Salt Lake City, 1999.

[2] A. Fasso', A. Ferrari, S. Roesler, P. R. Sala, G. Battistoni, F. Cerutti, E. Gadioli, M.V.Garzelli, F. Ballarini, A. Ottolenghi, A. Empl, and J. Ranft, "The physics models of FLUKA: status and recent developments", computing in High Energy and Nucler Physics 2003 conference (CHEP2003), La Jolla, CA, USA, March 24-28, 2003.

[3] A. Ferrari, P. R. Sala, A. Fasso', J. Ranft "Fluka: A multi-particle transport code"

[4] http://www.fluka.org 